\title{
GENERALIZED SOLUTION TO A NON LIPSCHITZ INTEGRAL EQUATION
}

\author{
VICTOR DÉVOUÉ \\ Communicated by Paul Eggermont
}

\begin{abstract}
We study a non Lipschitz integral equation in the framework of generalized functions. By means of suitable regularizations, we define an associated generalized problem. We obtain a generalized solution in an appropriate $(\mathcal{C}, \mathcal{E}, \mathcal{P})$ algebra. We compare the generalized solution with the classical one when it exists.
\end{abstract}

1. Introduction. Consider the integral equation

$$
(P): u(x)=\varphi(x)+\int_{0}^{x} F(x, t, u(t)) d t .
$$

We want to investigate solutions to this problem when the integrand $F$ is a smooth non Lipschitz function with respect to $u$ and the data $\varphi$ can be a distribution or other generalized function. So we search for solutions in algebras containing the space of distributions, which are invariant under nonlinear functions in addition. To do this, we use some regularization processes and cutoff techniques described in the framework of $(\mathcal{C}, \mathcal{E}, \mathcal{P})$-algebras of Marti [13-16]. These algebras, described in Section 2, are designed to admit multiparametric families of smooth functions as representatives of generalized functions.

There are a lot of numerical methods for integro-differential equations in a classical sense, when $F$ satisfies a Lipschitz condition [18]. In this paper we are interested in generalized solutions to integral equations with non-Lipschitz non linearity.

2010 AMS Mathematics subject classification. 35A01, 35A25, 35D99, 35R09, 45G10, 45K99, 46F30, 46T30

Keywords and phrases. Algebras of generalized functions, regularization of problems, regularization of data, non-linear generalized functions, non-linear problems, integral equations, non Lipschitz problems, non Lipschitz integral equations.

Received by the editors on October 21, 2012, and in revised form on February 11, 2013.

DOI:10.1216/JIE-2013-25-4-455 Copyright (C)2013 Rocky Mountain Mathematics Consortium 
The plan of this article is as follows. This section is followed by Section 2 which briefly introduces the generalized algebras with our application in mind. To generate this algebra we make some assumptions which depend on estimations closely related to the problem.

In Section 3, we associate to $(P)$ a well-formulated generalized problem $\left(P_{\text {gen }}\right)$ by means of a bi-parametric family of regularized Lipschitz problems

$$
\left(P_{(\varepsilon, \eta)}\right): u_{\varepsilon, \eta}(x)=\varphi_{\eta}(x)+\int_{0}^{x} F_{\varepsilon}\left(x, t, u_{\varepsilon, \eta}(t)\right) d t .
$$

The parameter $\varepsilon$ is used to render the problem Lipschitz and $\eta$ makes it regular. We replace $F$ with a family of Lipschitz functions $\left(F_{\varepsilon}\right)$ given by suitable cutoff techniques. Then, the classical successive approximation technique permits to obtain, for each $(\varepsilon, \eta)$, a global solution $u_{\varepsilon, \eta}$ to $\left(P_{(\varepsilon, \eta)}\right)$.

Notice that, because of the two regularization procedures and the estimates for this problem, Colombeau type algebras would be insufficient. Thus, we build a $(\mathcal{C}, \mathcal{E}, \mathcal{P})$-algebra, stable under the family $\left(F_{\varepsilon}\right)$, in which the class of the family $\left(u_{\varepsilon, \eta}\right)_{(\varepsilon, \eta)}$ is the expected generalized solution.

The solution depends a priori on the given cutoff family defining $\left(P_{g e n}\right)$; however, we show that this solution only depends on the class of the cutoff family of functions in some algebra.

Moreover, in Section 4, we show that if the initial problem $(P)$ admits a smooth solution $v$ satisfying appropriate growth estimates on some open subset $\Omega$ of $\mathbf{R}$, then this solution and the generalized one are equal in a meaning given in Theorem 24 .

2. Algebras of generalized functions. We recall briefly here the definition of the $(\mathcal{C}, \mathcal{E}, \mathcal{P})$-algebras upon which the remainder of this paper is based. We follow here the expositions found in [13-16]. We do not intend to properly define and explain $(\mathcal{C}, \mathcal{E}, \mathcal{P})$-algebras here; we rather want to fix the notations we will use in the latter sections. We refer the reader to the references.

The formalism described here, the $(\mathcal{C}, \mathcal{E}, \mathcal{P})$-algebras, is well suited for partial differential equations because of its parametric nature; this will become clear in the next section. 


\subsection{The presheaves of $(\mathcal{C}, \mathcal{E}, \mathcal{P})$-algebras.}

\subsubsection{Definitions. Take}

- $\Lambda$ a set of indices;

- $A$ a solid subring of the $\operatorname{ring} \mathbf{K}^{\Lambda},(\mathbf{K}=\mathbf{R}$ or $\mathbf{C})$, that is, $A$ has the following stability property: whenever $\left(\left|s_{\lambda}\right|\right)_{\lambda} \leq\left(r_{\lambda}\right)_{\lambda}$ (i.e., for any $\left.\lambda,\left|s_{\lambda}\right| \leq r_{\lambda}\right)$ for any pair $\left(\left(s_{\lambda}\right)_{\lambda},\left(r_{\lambda}\right)_{\lambda}\right) \in \mathbf{K}^{\Lambda} \times|A|$, it follows that $\left(s_{\lambda}\right)_{\lambda} \in A$, with $|A|=\left\{\left(\left|r_{\lambda}\right|\right)_{\lambda}:\left(r_{\lambda}\right)_{\lambda} \in A\right\}$;

- $I_{A}$ a solid ideal of $A$ with the same property;

- $\mathcal{E}$ a sheaf of $\mathbf{K}$-topological algebras on a topological space $X$, such that for any open set $\Omega$ in $X$, the algebra $\mathcal{E}(\Omega)$ is endowed with a family $\mathcal{P}(\Omega)=\left(p_{i}\right)_{i \in I(\Omega)}$ of seminorms satisfying that, for all $i \in I(\Omega)$, there exists

$$
(j, k, C) \in(I(\Omega))^{2} \times \mathbf{R}_{+}^{*},
$$

for all $f, g \in \mathcal{E}(\Omega)$,

$$
p_{i}(f g) \leq C p_{j}(f) p_{k}(g) \text {. }
$$

Assume that

- For any two open subsets $\Omega_{1}, \Omega_{2}$ of $X$ such that $\Omega_{1} \subset \Omega_{2}$, we have $I\left(\Omega_{1}\right) \subset I\left(\Omega_{2}\right)$ and, if $\rho_{1}^{2}$ is the restriction operator $\mathcal{E}\left(\Omega_{2}\right) \rightarrow \mathcal{E}\left(\Omega_{1}\right)$, then, for each $p_{i} \in \mathcal{P}\left(\Omega_{1}\right)$, the seminorm $\widetilde{p}_{i}=p_{i} \circ \rho_{1}^{2}$ extends $p_{i}$ to $\mathcal{P}\left(\Omega_{2}\right)$;

- For any family $\mathcal{F}=\left(\Omega_{h}\right)_{h \in H}$ of open subsets of $X$ if $\Omega=$ $\cup_{h \in H} \Omega_{h}$, then, for each $p_{i} \in \mathcal{P}(\Omega), i \in I(\Omega)$, there exists a finite subfamily $\Omega_{1}, \ldots, \Omega_{n(i)}$ of $\mathcal{F}$ and corresponding seminorms $p_{1} \in$ $\mathcal{P}\left(\Omega_{1}\right), \cdots, p_{n(i)} \in \mathcal{P}\left(\Omega_{n(i)}\right)$, such that, for each $u \in \mathcal{E}(\Omega)$,

$$
p_{i}(u) \leq p_{1}\left(u_{\mid \Omega_{1}}\right)+\cdots+p_{n(i)}\left(u_{\mid \Omega_{n(i)}}\right) .
$$

Set

$$
\begin{aligned}
\mathcal{X}_{(A, \mathcal{E}, \mathcal{P})}(\Omega) & =\left\{\left(u_{\lambda}\right)_{\lambda} \in[\mathcal{E}(\Omega)]^{\Lambda}: \forall i \in I(\Omega),\left(p_{i}\left(u_{\lambda}\right)\right)_{\lambda} \in|A|\right\}, \\
\mathbf{N}_{\left(I_{A}, \mathcal{E}, \mathcal{P}\right)}(\Omega) & =\left\{\left(u_{\lambda}\right)_{\lambda} \in[\mathcal{E}(\Omega)]^{\Lambda}: \forall i \in I(\Omega),\left(p_{i}\left(u_{\lambda}\right)\right)_{\lambda} \in\left|I_{A}\right|\right\}, \\
\mathcal{C} & =A / I_{A}
\end{aligned}
$$

One can prove that $\mathcal{X}_{(A, \mathcal{E}, \mathcal{P})}$ is a sheaf of subalgebras of the sheaf $\mathcal{E}^{\Lambda}$ and $\mathbf{N}_{\left(I_{A}, \mathcal{E}, \mathcal{P}\right)}$ is a sheaf of ideals of $\mathcal{X}_{(A, \mathcal{E}, \mathcal{P})}[\mathbf{1 4}]$. Moreover, the constant 
sheaf $\mathcal{X}_{(A, \mathbf{K},|.|)} / \mathbf{N}_{\left(I_{A}, \mathbf{K},|.|\right)}$ is exactly the sheaf $\mathcal{C}=A / I_{A}$, and if $\mathbf{K}=\mathbf{R}$, $\mathcal{C}$ will be denoted $\overline{\mathbf{R}}$.

Definition 1. We call the presheaf of $(\mathcal{C}, \mathcal{E}, \mathcal{P})$-algebra the factor presheaf of algebras over the $\operatorname{ring} \mathcal{C}=A / I_{A}$

$$
\mathcal{A}=\mathcal{X}_{(A, \mathcal{E}, \mathcal{P})} / \mathbf{N}_{\left(I_{A}, \mathcal{E}, \mathcal{P}\right)}
$$

We denote by $\left[u_{\lambda}\right]$ the class in $\mathcal{A}(\Omega)$ defined by the representative $\left(u_{\lambda}\right)_{\lambda \in \Lambda} \in \mathcal{X}_{(A, \mathcal{E}, \mathcal{P})}(\Omega)$.

2.1.2. Overgenerated rings. See [6]. Let $B_{p}=\left\{\left(r_{n, \lambda}\right)_{\lambda} \in\right.$ $\left.\left(\mathbf{R}_{+}^{*}\right)^{\Lambda}: n=1, \ldots, p\right\}$ and $B$ be the subset of $\left(\mathbf{R}_{+}^{*}\right)^{\Lambda}$ obtained as rational functions with coefficients in $\mathbf{R}_{+}^{*}$, of elements in $B_{p}$ as variables. Define

$$
A=\left\{\left(a_{\lambda}\right)_{\lambda} \in \mathbf{K}^{\Lambda}\left|\exists\left(b_{\lambda}\right)_{\lambda} \in B, \exists \lambda_{0} \in \Lambda, \forall \lambda \prec \lambda_{0}:\right| a_{\lambda} \mid \leq b_{\lambda}\right\} .
$$

Definition 2. In the above situation, we say that $A$ is overgenerated by $B_{p}$ (and it is easy to see that $A$ is a solid subring of $\mathbf{K}^{\Lambda}$ ). If $I_{A}$ is some solid ideal of $A$, we also say that $\mathcal{C}=A / I_{A}$ is overgenerated by $B_{p}$.

Example 3. For example, as a "canonical" ideal of $A$, we can take

$$
I_{A}=\left\{\left(a_{\lambda}\right)_{\lambda} \in \mathbf{K}^{\Lambda}\left|\forall\left(b_{\lambda}\right)_{\lambda} \in B, \exists \lambda_{0} \in \Lambda, \forall \lambda \prec \lambda_{0}:\right| a_{\lambda} \mid \leq b_{\lambda}\right\} .
$$

Remark 4 . We can see that with this definition $B$ is stable by inverse.

2.1.3. Relationship with distribution theory. Let $\Omega$ be an open subset of $\mathbf{R}^{n}$. The space of distributions $\mathcal{D}^{\prime}(\Omega)$ can be embedded into $\mathcal{A}(\Omega)$. If $\left(\theta_{\lambda}\right)_{\lambda \in(0,1]}$ is a family of mollifiers $\theta_{\lambda}(x)=1 / \lambda^{n} \theta(x / \lambda)$, $x \in \mathbf{R}^{n}, \int \theta(x) d x=1$ and if $T \in \mathcal{D}^{\prime}\left(\mathbf{R}^{n}\right)$, the convolution product family $\left(T * \theta_{\lambda}\right)_{\lambda}$ is a family of smooth functions slowly increasing in $1 / \lambda$. So, for $\Lambda=(0,1]$, we shall choose the subring $A$ overgenerated by some $B_{p}$ of $\left(\mathbf{R}_{+}^{*}\right)^{\Lambda}$ containing the family $(\lambda)_{\lambda},[\mathbf{4}, \mathbf{1 7}]$. 
2.1.4. The association process. We assume that $\Lambda$ is left-filtering for a given partial order relation $\prec$. We denote by $\Omega$ an open subset of $X, E$ a given sheaf of topological $\mathbf{K}$-vector spaces containing $\mathcal{E}$ as a subsheaf, $a$ a given map from $\Lambda$ to $\mathbf{K}$ such that $(a(\lambda))_{\lambda}=\left(a_{\lambda}\right)_{\lambda}$ is an element of $A$. We also assume that

$$
\mathbf{N}_{\left(I_{A}, \mathcal{E}, \mathcal{P}\right)}(\Omega) \subset\left\{\left(u_{\lambda}\right)_{\lambda} \in \mathcal{X}_{(A, \mathcal{E}, \mathcal{P})}(\Omega): \lim _{E(\Omega), \Lambda} u_{\lambda}=0\right\} .
$$

Definition 5. We say that $u=\left[u_{\lambda}\right]$ and $v=\left[v_{\lambda}\right] \in \mathcal{E}(\Omega)$ are $a-E$ associated if

$$
\lim _{E(\Omega), \Lambda} a_{\lambda}\left(u_{\lambda}-v_{\lambda}\right)=0 .
$$

That is to say, for each neighborhood $V$ of 0 for the $E$-topology, there exists $\lambda_{0} \in \Lambda$ such that $\lambda \prec \lambda_{0} \Rightarrow a_{\lambda}\left(u_{\lambda}-v_{\lambda}\right) \in V$. We write

$$
u \underset{E(\Omega)}{\stackrel{a}{\sim}} v
$$

Remark 6. We can also define an association process between $u=\left[u_{\lambda}\right]$ and $T \in \mathcal{E}(\Omega)$ by simply writing

$$
u \sim T \Longleftrightarrow \lim _{E(\Omega), \Lambda} u_{\lambda}=T .
$$

Taking $E=\mathcal{D}^{\prime}, \mathcal{E}=\mathrm{C}^{\infty}, \Lambda=(0,1]$, we recover the association process defined in the literature $([\mathbf{2}, \mathbf{3}])$.

2.1.5. $\mathcal{D}^{\prime}$-singular support. Assume that

$$
\mathbf{N}_{\mathcal{D}^{\prime}}^{\mathcal{A}}(\Omega)=\left\{\left(u_{\lambda}\right)_{\lambda} \in \mathcal{X}(\Omega): \lim _{\lambda \rightarrow 0} u_{\lambda}=0 \text { in } \mathcal{D}^{\prime}(\Omega)\right\} \supset \mathbf{N}(\Omega) .
$$

Set

$$
\mathcal{D}_{\mathcal{A}}^{\prime}(\Omega)=\left\{\left[u_{\lambda}\right] \in \mathcal{A}(\Omega): \exists T \in \mathcal{D}^{\prime}(\Omega), \lim _{\lambda \rightarrow 0}\left(u_{\lambda}\right)=T \text { in } \mathcal{D}^{\prime}(\Omega)\right\} .
$$

$\mathcal{D}_{\mathcal{A}}^{\prime}(\Omega)$ is clearly well defined because the limit is independent of the chosen representative; indeed, if $\left(i_{\lambda}\right)_{\lambda} \in \mathbf{N}(\Omega)$, we have $\lim _{\lambda \rightarrow 0, \mathcal{D}^{\prime}(\mathbf{R})} i_{\lambda}=$ 0 . 
$\mathcal{D}_{\mathcal{A}}^{\prime}(\Omega)$ is an $\mathbf{R}$-vector subspace of $\mathcal{A}(\Omega)$. Therefore, we can consider the set $\mathcal{O}_{D_{\mathcal{A}}^{\prime}}$ of all $x$ having a neighborhood $V$ on which $u$ is associated to a distribution:

$$
\mathcal{O}_{D_{\mathcal{A}}^{\prime}}(u)=\left\{x \in \Omega: \exists V \in \mathcal{V}(x),\left.u\right|_{V} \in \mathcal{D}_{\mathcal{A}}^{\prime}(V)\right\},
$$

$\mathcal{V}(x)$ being the set of all neighborhoods of $x$.

Definition 7. The $\mathcal{D}^{\prime}$-singular support of $u \in \mathcal{A}(\Omega)$, denoted $\operatorname{singsupp}_{\mathcal{D}^{\prime}}(u)=S_{\mathcal{D}_{\mathcal{A}}^{\prime}}^{\mathcal{A}}(u)$, is the set

$$
S_{\mathcal{D}_{\mathcal{A}^{\prime}}}^{\mathcal{A}}(u)=\Omega \backslash \mathcal{O}_{D_{\mathcal{A}}^{\prime}}(u) .
$$

2.2. Algebraic framework for our problem. Set $\mathcal{E}=\mathrm{C}^{\infty}$, $X=\mathbf{R}^{d}$ for $d=1,2, E=\mathcal{D}^{\prime}$ and $\Lambda$ a set of indices, $\lambda \in \Lambda$. For any open set $\Omega$, in $\mathbf{R}^{d}, \mathcal{E}(\Omega)$ is endowed with the $\mathcal{P}(\Omega)$ topology of uniform convergence of all derivatives on compact subsets of $\Omega$. This topology may be defined by the family of the seminorms

$$
P_{K, l}\left(u_{\lambda}\right)=\sup _{|\alpha| \leq l} P_{K, \alpha}\left(u_{\lambda}\right) \text { with } P_{K, \alpha}\left(u_{\lambda}\right)=\sup _{x \in K}\left|D^{\alpha} u_{\lambda}(x)\right|, K \Subset \Omega,
$$

where the notation $K \Subset \mathbf{R}^{2}$ means that $K$ is a compact subset of $\mathbf{R}^{2}$ and $D^{\alpha}=\partial^{\alpha_{1}+\cdots+\alpha_{d}} / \partial z_{1}^{\alpha_{1}} \cdots \partial z_{d}^{\alpha_{d}}$ for $z=\left(z_{1}, \ldots, z_{d}\right) \in \Omega, l \in \mathbf{N}$, $\alpha=\left(\alpha_{1}, \ldots, \alpha_{d}\right) \in \mathbf{N}^{d}$. Let $A$ be a subring of the ring $\mathbf{R}^{\Lambda}$ of family of reals with the usual laws. We consider a solid ideal $I_{A}$ of $A$. Then we have

$$
\begin{aligned}
& \mathcal{X}(\Omega)=\left\{\left(u_{\lambda}\right)_{\lambda} \in\left[\mathrm{C}^{\infty}(\Omega)\right]^{\Lambda}: \forall K \Subset \Omega, \forall l \in \mathbf{N},\left(P_{K, l}\left(u_{\lambda}\right)\right)_{\lambda} \in|A|\right\}, \\
& \mathbf{N}(\Omega)=\left\{\left(u_{\lambda}\right)_{\lambda} \in\left[\mathrm{C}^{\infty}(\Omega)\right]^{\Lambda}: \forall K \Subset \Omega, \forall l \in \mathbf{N},\left(P_{K, l}\left(u_{\lambda}\right)\right)_{\lambda} \in\left|I_{A}\right|\right\}, \\
& \mathcal{A}(\Omega)=\mathcal{X}(\Omega) / \mathbf{N}(\Omega) .
\end{aligned}
$$

The generalized derivation $D^{\alpha}: u\left(=\left[u_{\varepsilon}\right]\right) \mapsto D^{\alpha} u=\left[D^{\alpha} u_{\varepsilon}\right]$ provides $\mathcal{A}(\Omega)$ with a differential algebraic structure.

Example 8. Set $\Lambda=(0,1]$. Consider

$$
\begin{aligned}
A=\left\{\left(m_{\lambda}\right)_{\lambda} \in \mathbf{R}^{\Lambda}: \exists p \in \mathbf{R}_{+}^{*}, \exists C \in \mathbf{R}_{+}^{*}\right. & , \exists \mu \in(0,1], \\
& \left.\forall \lambda \in(0, \mu],\left|m_{\lambda}\right| \leq C \lambda^{-p}\right\}
\end{aligned}
$$


and the ideal

$$
\begin{aligned}
I_{A}=\left\{\left(m_{\lambda}\right)_{\lambda} \in \mathbf{R}^{\Lambda}: \forall q \in \mathbf{R}_{+}^{*}, \exists D \in \mathbf{R}_{+}^{*}\right. & , \exists \mu \in(0,1], \\
& \left.\forall \lambda \in(0, \mu],\left|m_{\varepsilon}\right| \leq D \lambda^{q}\right\} .
\end{aligned}
$$

In this case the sheaf of factor algebras $\mathcal{G}(\cdot)=\mathcal{X}(\cdot) / \mathbf{N}(\cdot)$ is called the sheaf of simplified Colombeau algebras and $\mathcal{G}\left(\mathbf{R}^{d}\right)$ is the simplified Colombeau algebra of generalized functions $[\mathbf{2}, \mathbf{3}]$.

We have the analogue of Theorem 1.2 .3 of $[\mathbf{1}]$ for $(\mathcal{C}, \mathcal{E}, \mathcal{P})$-algebras. We suppose here that $\Lambda$ is left filtering and give this proposition for $\mathcal{A}(\mathbf{R})$, although it is valid in more general situations.

Proposition 9. Let $B$ be the set introduced in Definition 2, and assume that there exists an $\left(a_{\lambda}\right)_{\lambda} \in B$ with $\lim _{\lambda \rightarrow 0} a_{\lambda}=0$. Consider $\left(u_{\lambda}\right)_{\lambda} \in \mathcal{X}(\mathbf{R})$ such that, for all $K \Subset \mathbf{R}^{2}$,

$$
\left(P_{K, 0}\left(u_{\lambda}\right)\right)_{\lambda} \in\left|I_{A}\right| \text {. }
$$

Then $\left(u_{\lambda}\right)_{\lambda} \in \mathbf{N}(\mathbf{R})$.

We refer the reader to [6] for a similar proof.

2.2.1. Integration of generalized functions. A generalized function $u$ can be integrated over a compact set $K$ of $\Omega$ defining $\int_{K} u(y) d y$ as the generalized number with representative $\left(\int_{K} u_{\lambda}(y) d y\right)_{\lambda}$. (This class does not depend on the choice of the representative of $u$.)

The integral of a generalized function having a compact support is an immediate extension of the previous case; we set $\int_{\Omega} u(y) d y=$ $\int_{K} u(y) d y$ where $K$ is any compact set containing supp $u$ in its interior. See $[\mathbf{1}, \mathbf{1 0}]$.

\subsubsection{Generalized operator associated to a stability prop- erty.}

Definition 10. Let $\Omega$ be an open subset of $\mathbf{R}$ and $F \in \mathrm{C}^{\infty}\left(\Omega^{2} \times\right.$ $\mathbf{R}, \mathbf{R})$. We say that the algebra $\mathcal{A}(\Omega)$ is stable under $F$ if for all 
$\left(u_{\varepsilon}\right)_{\varepsilon} \in \mathcal{X}(\Omega)$ and $\left(i_{\varepsilon}\right)_{\varepsilon} \in \mathbf{N}(\Omega)$, we have $\left(F\left(\cdot, \cdot, u_{\varepsilon}\right)\right)_{\varepsilon} \in \mathcal{X}(\Omega)$; $\left(F\left(\cdot, \cdot, u_{\varepsilon}+i_{\varepsilon}\right)-F\left(\cdot, \cdot, u_{\varepsilon}\right)\right)_{\varepsilon} \in \mathbf{N}(\Omega)$.

If $\mathcal{A}(\mathbf{R})$ is stable under $F$, for $u=\left[u_{\varepsilon}\right] \in \mathcal{A}(\mathbf{R}),\left[F\left(\cdot, \cdot, u_{\varepsilon}\right)\right]$ is a welldefined element of $\mathcal{A}$ (i.e., not depending on the representative $\left(u_{\varepsilon}\right)_{\varepsilon}$ of $u)$.

Definition 11. Let $\Omega$ be an open subset of $\mathbf{R}$ and $F \in \mathrm{C}^{\infty}\left(\Omega^{2} \times\right.$ $\mathbf{R}, \mathbf{R})$. We say that $F$ is smoothly tempered if the following two conditions are satisfied:

For each $K \Subset \mathbf{R}, l \in \mathbf{N}$ and $u \in \mathrm{C}^{\infty}(\Omega, \mathbf{R})$, there is a positive finite sequence $C_{0}, \ldots, C_{l}$, such that

$$
P_{K^{2}, l}(F(\cdot, \cdot, u)) \leq \sum_{i=0}^{l} C_{i}\left(P_{K, l}(u)\right)^{i}
$$

For each $K \Subset \mathbf{R}, l \in \mathbf{N}, u, v \in \mathrm{C}^{\infty}(\Omega, \mathbf{R})$, there is a positive finite sequence $D_{1}, \ldots, D_{l}$ such that

$$
P_{K^{2}, l}(F(\cdot, \cdot, v)-F(\cdot, \cdot, u)) \leq \sum_{j=1}^{l} D_{j}\left(P_{K, l}(v-u)\right)^{j}
$$

We then have the following obvious proposition:

Proposition 12. Let $\Omega$ be an open subset of $\mathbf{R}$ and $F \in \mathrm{C}^{\infty}\left(\Omega^{2} \times\right.$ $\mathbf{R}, \mathbf{R})$. Assume that $F$ is smoothly tempered. Then $\mathcal{A}(\mathbf{R})$ is stable under $F$.

This leads to the following:

Definition 13. If $\mathcal{A}(\mathbf{R})$ is stable under $F$, the operator

$$
\mathcal{F}: \mathcal{A}(\mathbf{R}) \longrightarrow \mathcal{A}(\mathbf{R}), \quad u=\left[u_{\varepsilon}\right] \longmapsto\left[F\left(\cdot, \cdot, u_{\varepsilon}\right)\right]
$$

is called the generalized operator associated to $F$. See $[\mathbf{6}]$. 
3. Existence of solutions to the problem in $(\mathcal{C}, \mathcal{E}, \mathcal{P})$-algebras. We study the following problem:

$$
(P): u(x)=\varphi(x)+\int_{0}^{x} F(x, t, u(t)) d t
$$

where $\varphi$ is the data which will be specified later. The function $F$ may be non Lipschitz with respect to $u$.

Let $(\varepsilon, \eta) \in(0,1]^{2}$. As the data $\varphi$ is as irregular as distribution, we set $\varphi_{\eta}=\varphi * \theta_{\eta}$ and $\varphi=\left[\varphi_{\eta}\right]$ where $\left(\theta_{\eta}\right)_{\eta}$ is a chosen family of mollifiers.

3.1. Cut off procedure. Let $\varepsilon$ be a parameter belonging to the interval $(0,1],\left(r_{\varepsilon}\right)_{\varepsilon}$ be in $\left(\mathbf{R}_{*}^{+}\right)^{(0,1]}$ such that $\lim _{\varepsilon \rightarrow 0} r_{\varepsilon}=+\infty$. Consider a family of smooth one-variable functions $\left(g_{\varepsilon}\right)_{\varepsilon}$ such that $\sup _{z \in\left[-r_{\varepsilon}, r_{\varepsilon}\right]}\left|g_{\varepsilon}(z)\right|=1$ with

$$
g_{\varepsilon}(z)= \begin{cases}0 & \text { if }|z| \geq r_{\varepsilon} \\ 1 & \text { if }-r_{\varepsilon}+1 \leq z \leq r_{\varepsilon}-1\end{cases}
$$

Assume that $\partial^{n} g_{\varepsilon} / \partial z^{n}$ is bounded on $\left[-r_{\varepsilon}, r_{\varepsilon}\right]$ for any integer $n, n>0$ and

$$
\sup _{z \in\left[-r_{\varepsilon}, r_{\varepsilon}\right]}\left|\frac{\partial^{n} g_{\varepsilon}}{\partial z^{n}}(z)\right|=M_{n}
$$

Let $\phi_{\varepsilon}(z)=z g_{\varepsilon}(z)$. We approximate the function $F$ by

$$
(x, y, z) \longmapsto F\left(x, y, \phi_{\varepsilon}(z)\right)=F_{\varepsilon}(x, y, z)
$$

then problem $(P)$ is changed into the family of regularized Lipschitz problems

$$
\left(P_{(\varepsilon, \eta)}\right): u_{\varepsilon, \eta}(x)=\varphi_{\eta}(x)+\int_{0}^{x} F_{\varepsilon}\left(x, t, u_{\varepsilon, \eta}(t)\right) d t .
$$

Here $F$ can be non-Lipschitz but still have polynomial growth. More precisely, we assume the existence of $p \in \mathbf{N}$ such that, for all $K \Subset \mathbf{R}$ and for all $l \in \mathbf{N}$, there exists a $\mu_{K, l}>0$,

$$
\sup _{(x, t, z) \in K^{2} \times \mathbf{R}}\left|D^{l} F(x, t, z)\right| \leq \mu_{K, l}(1+|z|)^{p} .
$$


Thus,

$$
\begin{aligned}
\sup _{(x, t, z) \in K^{2} \times \mathbf{R} ;|\alpha| \leq l}\left|D^{l} F_{\varepsilon}(x, t, z)\right| & =\sup _{(x, t) \in K^{2} ;|z| \leq r_{\varepsilon} ;|\alpha| \leq l}\left|D^{l} F\left(x, t, \phi_{\varepsilon}(z)\right)\right| \\
& \leq \mu_{K, l}\left(1+r_{\varepsilon}\right)^{p} .
\end{aligned}
$$

In particular, we set

$$
m_{K, \varepsilon}=\sup _{(x, t) \in K^{2} ; z \in \mathbf{R}}\left|\frac{\partial}{\partial z} F_{\varepsilon}(x, t, z)\right| \leq \mu_{K, 1}\left(1+r_{\varepsilon}\right)^{p} .
$$

Theorem 14. Set $n \in \mathbf{N}$. Let $\left(F_{\varepsilon}\right)_{\varepsilon} \in\left(\mathrm{C}^{\infty}\left(\mathbf{R}^{3}, \mathbf{R}\right)\right)^{(0,1]}$. Assume that for all $\varepsilon \in(0,1]$ and for all $(x, t) \in \mathbf{R}^{2}$,

$$
F_{\varepsilon}(x, t, 0)=0,
$$

for all $\varepsilon \in(0,1]$, there exists $p>0$, for all $n \in \mathbf{N}$ and for all $K \Subset \mathbf{R}$, there exists $\mu_{K, n}>0$,

$$
\sup _{(x, t) \in K^{2} ; z \in \mathbf{R} ;|\alpha| \leq l}\left|D^{n} F_{\varepsilon}(x, t, z)\right| \leq \mu_{K, n}\left(1+r_{\varepsilon}\right)^{p},
$$

then $\mathcal{A}(\mathbf{R})$ is stable under the family $\left(F_{\varepsilon}\right)_{\varepsilon}$.

See $[\mathbf{7}, \mathbf{8}]$ for a similar proof.

3.2. Construction of $\mathcal{A}(\mathbf{R})$. We make the following assumptions to generate a convenient $(\mathcal{C}, \mathcal{E}, \mathcal{P})$-algebra adapted to our problem: for all $K \Subset \mathbf{R}$ and for all $l \in \mathbf{N}$, there exists $\mu_{K, l}>0$,

$\left(H_{1}\right)$

$$
\sup _{(x, t, z) \in K^{2} \times \mathbf{R}, l}\left|D^{l} F(x, t, z)\right| \leq \mu_{K, l}(1+|z|)^{p} .
$$

$\left(H_{2}\right) \varphi_{\eta} \in \mathrm{C}^{\infty}(\mathbf{R})$

$\left(H_{3}\right)\left\{\begin{array}{c}\mathcal{C}=A / I_{A} \text { is overgenerated by the following } \\ \quad \text { elements of } \mathbf{R}_{*}^{(0,1]} \\ (\varepsilon)_{\varepsilon, \eta},(\eta)_{\varepsilon, \eta},\left(r_{\varepsilon}\right)_{\varepsilon, \eta},\left(\exp r_{\varepsilon}\right)_{\varepsilon, \eta} .\end{array}\right.$

$\left(H_{4}\right)\left\{\begin{array}{l}\mathcal{A}(\mathbf{R})=\mathcal{X}(\mathbf{R} / \mathbf{N}(\mathbf{R})) \text { is built on } \mathcal{C} \text { with } \\ (\mathcal{E}, \mathcal{P})=\left(\mathrm{C}^{\infty}(\mathbf{R}),\left(P_{K, l}\right)_{K \Subset \mathbf{R}^{2}, l \in \mathbf{N}}\right) .\end{array}\right.$ 
Then the data $\varphi$ belong to $\mathcal{A}(\mathbf{R})$ and $u$ is searched in the algebra $\mathcal{A}(\mathbf{R})$.

\subsection{A generalized differential problem associated to the} classical one. The problem associated to $(P)$ can be written as the well-formulated one

$$
\left(P_{\text {gen }}\right): u(x)=\varphi(x)+\int_{0}^{x} \mathcal{F}(u)(t) d t
$$

where $u$ is searched in the algebra $\mathcal{A}(\mathbf{R})$ and $\mathcal{F}$ is defined as previously.

The family of problems

$$
\left(P_{(\varepsilon, \eta)}\right): u_{\varepsilon, \eta}(x)=\varphi_{\eta}(x)+\int_{0}^{x} F_{\varepsilon}\left(x, t, u_{\varepsilon, \eta}(t)\right) d t
$$

is associated to problem $(P)$.

In terms of representatives, and thanks to the stability hypothesis, solving the generalized problem $\left(P_{g e n}\right)$ amounts to finding a family $\left(u_{\varepsilon, \eta}\right)_{\varepsilon, \eta} \in \mathcal{X}(\mathbf{R})$ such that

$$
u_{\varepsilon, \eta}(x)-\left(\varphi_{\eta}(x)+i_{\varepsilon, \eta}(x)\right)=\int_{0}^{x} F_{\varepsilon}\left(x, t, u_{\varepsilon, \eta}(t)\right) d t+j_{\varepsilon, \eta}(x),
$$

where $\left(j_{\varepsilon}\right)_{\varepsilon},\left(i_{\varepsilon}\right)_{\varepsilon} \in \mathbf{N}(\mathbf{R})$.

Suppose we can find $u_{\varepsilon, \eta} \in \mathrm{C}^{\infty}(\mathbf{R})$ verifying

$$
\left(P_{(\varepsilon, \eta)}\right): u_{\varepsilon, \eta}(x)=\varphi_{\eta}(x)+\int_{0}^{x} F_{\varepsilon}\left(x, t, u_{\varepsilon, \eta}(t)\right) d t
$$

then, if we can prove that $\left(u_{\varepsilon, \eta}\right)_{\varepsilon, \eta} \in \mathcal{X}(\mathbf{R}), u=\left[u_{\varepsilon, \eta}\right]$ is a solution of $\left(P_{\text {gen }}\right)$.

3.4. Estimates for a parametrized regular problem. We are going to replace $(P)$ with a family $\left(P_{(\varepsilon, \eta)}\right)$ of regularized problems:

$$
\left(P_{(\varepsilon, \eta)}\right): u_{\varepsilon, \eta}(x)=\varphi_{\eta}(x)+\int_{0}^{x} F_{\varepsilon}\left(x, t, u_{\varepsilon, \eta}(t)\right) d t,
$$


where $F_{\varepsilon}$ is Lipschitz, $\varphi_{\eta}$ regular, we are going to prove that $\left(P_{(\varepsilon, \eta)}\right)$ has a unique smooth solution under the following assumption

$$
\left(H_{\varepsilon, \eta}\right)\left\{\begin{array}{l}
F_{\varepsilon} \in \mathrm{C}^{\infty}\left(\mathbf{R}^{3}, \mathbf{R}\right), \forall K \Subset \mathbf{R}, \\
\quad \sup _{(x, y) \in K^{2} ; z \in \mathbf{R}}\left|\partial_{z} F_{\varepsilon}(x, y, z)\right|=m_{K, \varepsilon}<+\infty ; \\
\varphi_{\eta} \in \mathrm{C}^{\infty}(\mathbf{R}) .
\end{array}\right.
$$

Theorem 15. Under Assumption $\left(H_{\varepsilon, \eta}\right)$, Problem $\left(P_{(\varepsilon, \eta)}\right)$ has a unique solution in $C^{\infty}(\mathbf{R})$.

The main idea consists in a Picard's procedure to define a sequence of successive approximations

$$
u_{n, \varepsilon, \eta}(x)=u_{0, \varepsilon, \eta}(x)+\int_{0}^{x} F_{\varepsilon}\left(x, t, u_{n-1, \varepsilon, \eta}(t)\right) d t,
$$

where $u_{0, \varepsilon, \eta}(x)=\varphi_{\eta}(x)$. For any compact subset $K$ of $\mathbf{R}$ there exits a compact subset $K_{\lambda}=[-\lambda, \lambda] \Subset \mathbf{R}$ containing $K$.

Putting $v_{n, \varepsilon, \eta}=u_{n, \varepsilon, \eta}-u_{n-1, \varepsilon, \eta}, m_{\lambda, \varepsilon}=m_{K_{\lambda}, \varepsilon}$, we prove that

$$
\left\|v_{n, \varepsilon, \eta}\right\|_{\infty, K_{\lambda}} \leq \frac{\Phi_{\lambda, \varepsilon, \eta}}{m_{\lambda, \varepsilon}} \frac{\left(\lambda m_{\lambda, \varepsilon}\right)^{n}}{n !}
$$

where

$$
\Phi_{\lambda, \varepsilon, \eta}=\sup _{(x, t) \in K_{\lambda}^{2}}\left|F_{\varepsilon}(x, t, 0)\right|+m_{\lambda, \varepsilon} \sup _{x \in K_{\lambda}}\left|\varphi_{\eta}(x)\right| .
$$

Finally, the sequence $u_{n, \varepsilon, \eta}$ converges uniformly on any compact set to

$$
u_{\varepsilon, \eta}=u_{0, \varepsilon, \eta}+\sum_{n \geq 1} v_{n, \varepsilon, \eta},
$$

which is the solution to $\left(P_{(\varepsilon, \eta)}\right)$. Gronwall's lemma gives the uniqueness of $u_{\varepsilon, \eta}$. Moreover, we have the estimate

$$
\left\|u_{\varepsilon, \eta}\right\|_{\infty, K} \leq\left\|u_{\varepsilon, \eta}\right\|_{\infty, K_{\lambda}} \leq\left\|u_{0, \varepsilon, \eta}\right\|_{\infty, K_{\lambda}}+\frac{\Phi_{\lambda, \varepsilon, \eta}}{m_{\lambda, \varepsilon}} \exp \left(\lambda m_{\lambda, \varepsilon}\right) .
$$

These results are proved in Appendix 5. 


\subsection{Solution to $\left(P_{g e n}\right)$.}

Proposition 16. The family $\left(u_{\varepsilon, \eta}\right)_{\varepsilon, \eta}$ is a representative of a generalized function $u$ which belongs to the algebra $\mathcal{A}(\mathbf{R})$. Then, $\left(P_{\text {gen }}\right)$ admits $u=\left[u_{\varepsilon, \eta}\right]_{\mathcal{A}(\mathbf{R})}$ as a solution.

Proof. Take $K$ to be a compact subset of $\mathbf{R}$. There exists a compact subset $K_{\lambda}=[-\lambda, \lambda] \Subset \mathbf{R}$ containing $K$ such that

$$
\left\|u_{\varepsilon, \eta}\right\|_{\infty, K} \leq\left\|u_{\varepsilon, \eta}\right\|_{\infty, K_{\lambda}} \leq\left\|u_{0, \varepsilon, \eta}\right\|_{\infty, K_{\lambda}}+\frac{\Phi_{\lambda, \varepsilon, \eta}}{m_{\lambda, \varepsilon}} \exp \left(\lambda m_{\lambda, \varepsilon}\right)
$$

where $m_{\lambda, \varepsilon}=m_{K_{\lambda}, \varepsilon}$,

$$
\Phi_{\lambda, \varepsilon, \eta}=\sup _{(x, t) \in K_{\lambda}^{2}}\left|F_{\varepsilon}(x, t, 0)\right|+m_{\lambda, \varepsilon} \sup _{x \in K_{\lambda}}\left|\varphi_{\eta}(x)\right| .
$$

So

$\frac{\Phi_{\lambda, \varepsilon, \eta}}{m_{\lambda, \varepsilon}} \exp \left(\lambda m_{\lambda, \varepsilon}\right)=\frac{\left\|F_{\varepsilon}(\cdot, \cdot, 0)\right\|_{\infty, K_{\lambda}^{2}}}{m_{\lambda, \varepsilon}} \exp \left(\lambda m_{\lambda, \varepsilon}\right)+\left\|\varphi_{\eta}\right\|_{\infty, K_{\lambda}} \exp \left(\lambda m_{\lambda, \varepsilon}\right)$.

Consequently,

$$
\begin{aligned}
\left\|u_{\varepsilon, \eta}\right\|_{\infty, K} \leq & \left\|u_{\varepsilon, \eta}\right\|_{\infty, K_{\lambda}} \\
\leq & \left\|\varphi_{\eta}\right\|_{\infty, K_{\lambda}}\left(1+\exp \left(\lambda m_{\lambda, \varepsilon}\right)\right) \\
& +\frac{\left\|F_{\varepsilon}(\cdot, \cdot, 0)\right\|_{\infty, K_{\lambda}^{2}}}{m_{\lambda, \varepsilon}} \exp \left(\lambda m_{\lambda, \varepsilon}\right)
\end{aligned}
$$

thus

(VD1)

$$
P_{K_{\lambda}, 0}\left(u_{\varepsilon, \eta}\right) \leq c_{1, \lambda, \varepsilon}+c_{2, \lambda, \varepsilon} P_{K_{\lambda}, 0}\left(\varphi_{\eta}\right),
$$

where the constants

$$
\left.c_{1, \lambda, \varepsilon}=\frac{\left\|F_{\varepsilon}(\cdot, \cdot, 0)\right\|_{\infty, K_{\lambda}^{2}}}{m_{\lambda, \varepsilon}} \exp \left(\lambda m_{\lambda, \varepsilon}\right)\right)
$$


and $c_{2, \lambda, \varepsilon}=\left(1+\exp \left(\lambda m_{\lambda, \varepsilon}\right)\right)$ depend only on $F_{\varepsilon}$ and $K_{\lambda}$. So $\left(P_{K_{\lambda}, 0}\left(u_{\varepsilon, \eta}\right)\right)_{\varepsilon, \eta} \in|A|$. For any $(x, t) \in K_{\lambda}^{2}$, the mean value theorem yields the expression

$$
F_{\varepsilon}(x, t, z)-F_{\varepsilon}(x, t, r)=(z-r) \int_{0}^{1} \frac{\partial F_{\varepsilon}}{\partial z}(x, t, r+\sigma(t-r)) d \sigma .
$$

In particular,

$$
F_{\varepsilon}\left(x, t, u_{\varepsilon, \eta}(t)\right)=F_{\varepsilon}(x, t, 0)+u_{\varepsilon, \eta}(t) \int_{0}^{1} \frac{\partial F_{\varepsilon}}{\partial z}\left(x, t, \sigma\left(u_{\varepsilon, \eta}(t)\right)\right) d \sigma .
$$

So

$$
\begin{aligned}
\left|F_{\varepsilon}\left(x, t, u_{\varepsilon, \eta}(t)\right)\right| & \left.\leq \mid F_{\varepsilon}(x, t, 0)\right) \mid+m_{\lambda, \varepsilon}\left\|u_{\varepsilon, \eta}\right\|_{\infty, K_{\lambda}} \\
& \leq\left\|F_{\varepsilon}(\cdot, \cdot, 0)\right\|_{\infty, K_{\lambda}^{2}}+m_{\lambda, \varepsilon}\left\|u_{\varepsilon, \eta}\right\|_{\infty, K_{\lambda}} .
\end{aligned}
$$

Then, according to (VD1),

$$
\left|F_{\varepsilon}\left(x, t, u_{\varepsilon, \eta}(t)\right)\right| \leq\left\|F_{\varepsilon}(\cdot, \cdot, 0)\right\|_{\infty, K_{\lambda}^{2}}+m_{\lambda, \varepsilon}\left(c_{1, \lambda, \varepsilon}+c_{2, \lambda, \varepsilon} P_{K_{\lambda}, 0}\left(\varphi_{\eta}\right)\right) .
$$

Finally,

$$
P_{K_{\lambda}^{2}, 0}\left(F_{\varepsilon}\left(\cdot, \cdot, u_{\varepsilon, \eta}\right)\right) \leq c_{3, \lambda, \varepsilon}+c_{4, \lambda, \varepsilon} P_{K_{\lambda}, 0}\left(\varphi_{\eta}\right),
$$

with $c_{3, \lambda, \varepsilon}=\left\|F_{\varepsilon}(\cdot, \cdot, 0)\right\|_{\infty, K_{\lambda}^{2}}+m_{\lambda, \varepsilon} c_{1, \lambda, \varepsilon}$ and $c_{4, \lambda, \varepsilon}=m_{\lambda, \varepsilon} c_{2, \lambda, \varepsilon}$. We have

$$
\frac{\partial u_{\varepsilon, \eta}}{\partial x}(x)=\frac{\partial \varphi_{\eta}}{\partial x}(x)+F_{\varepsilon}\left(x, x, u_{\varepsilon, \eta}(x)\right)+\int_{0}^{x} \frac{\partial F_{\varepsilon}}{\partial x}\left(x, t, u_{\varepsilon, \eta}(t)\right) d t ;
$$

thus,

$$
\left|\frac{\partial u_{\varepsilon, \eta}}{\partial x}(x)\right| \leq P_{K_{\lambda}, 1}\left(\varphi_{\eta}\right)+P_{K_{\lambda}^{2}, 0}\left(F_{\varepsilon}\right)+\int_{0}^{x} \mu_{K_{\lambda}, 1}\left(1+r_{\varepsilon}\right)^{p} d t .
$$

Then

$$
\begin{aligned}
P_{K_{\lambda}, 1}\left(u_{\varepsilon, \eta}\right) & \leq P_{K_{\lambda}, 1}\left(\varphi_{\eta}\right)+P_{K_{\lambda}^{2}, 0}\left(F_{\varepsilon}\right)+\lambda \mu_{K_{\lambda}, 1}\left(1+r_{\varepsilon}\right)^{p} \\
& \leq P_{K_{\lambda}, 1}\left(\varphi_{\eta}\right)+c_{3, \lambda, \varepsilon}+c_{4, \lambda, \varepsilon} P_{K_{\lambda}, 0}\left(\varphi_{\eta}\right)+\lambda \mu_{K_{\lambda}, 1}\left(1+r_{\varepsilon}\right)^{p} .
\end{aligned}
$$


We have

$$
\begin{aligned}
\frac{\partial^{n} u_{\varepsilon, \eta}}{\partial x^{n}}(x)= & \frac{\partial^{n} \varphi_{\eta}}{\partial x^{n}}(x)+n \frac{\partial^{n-1}}{\partial x^{n-1}} F_{\varepsilon}\left(x, x, u_{\varepsilon, \eta}(x)\right) \\
& +\int_{0}^{x} \frac{\partial^{n} F_{\varepsilon}}{\partial x^{n}}\left(x, t, u_{\varepsilon, \eta}(t)\right) d t .
\end{aligned}
$$

Then

$$
\begin{aligned}
P_{K_{\lambda}, n}\left(u_{\varepsilon, \eta}\right) & \leq P_{K_{\lambda}, n}\left(\varphi_{\eta}\right)+n P_{K_{\lambda}^{2}, n-1}\left(F_{\varepsilon}\right)+\lambda P_{K_{\lambda}, n}\left(F_{\varepsilon}\right) \\
& \leq P_{K_{\lambda}, n}\left(\varphi_{\eta}\right)+n \mu_{K_{\lambda}, n-1}\left(1+r_{\varepsilon}\right)^{p}+\lambda \mu_{K_{\lambda}, n}\left(1+r_{\varepsilon}\right)^{p} \\
& \leq P_{K_{\lambda}, n}\left(\varphi_{\eta}\right)+\left(n \mu_{K_{\lambda}, n-1}+\lambda \mu_{K_{\lambda}, n}\right)\left(1+r_{\varepsilon}\right)^{p} .
\end{aligned}
$$

We deduce that $\left(P_{K_{\lambda}, n}\left(u_{\varepsilon, \eta}\right)\right)_{\varepsilon, \eta} \in A$ and then $\left(u_{\varepsilon, \eta}\right)_{\varepsilon, \eta} \in \mathcal{A}(\mathbf{R})$. Let $v$ be another solution to $\left(P_{g e n}\right)$ with $v=\left(v_{\varepsilon, \eta}\right)_{\varepsilon, \eta}$. For any $x \in K_{\lambda}$, we have

$$
v_{\varepsilon, \eta}(x)=i_{\eta}(x)+\varphi_{\eta}(x)+\int_{0}^{x} F_{\varepsilon}\left(x, t, v_{\varepsilon, \eta}(t)\right) d t .
$$

Set $w_{\varepsilon, \eta}=\left(v_{\varepsilon, \eta}-u_{\varepsilon, \eta}\right)$. Then

$$
w_{\varepsilon, \eta}(x)=i_{\eta}(x)+\int_{0}^{x}\left(F_{\varepsilon}\left(x, t, v_{\varepsilon, \eta}(t)\right)-F_{\varepsilon}\left(x, t, u_{\varepsilon, \eta}(t)\right) d t,\right.
$$

but

$$
\begin{aligned}
F_{\varepsilon}\left(x, t, v_{\varepsilon, \eta}(t)\right)-F_{\varepsilon} & \left(x, t, u_{\varepsilon, \eta}(t)\right) \\
& =w_{\varepsilon, \eta}(t) \int_{0}^{1} \frac{\partial F_{\varepsilon}}{\partial z}\left(x, t, u_{\varepsilon, \eta}(t)+\theta w_{\varepsilon, \eta}(t)\right) \mathrm{d} \theta,
\end{aligned}
$$

so

$$
w_{\varepsilon, \eta}(x)=i_{\eta}(x)+\int_{0}^{x}\left(w_{\varepsilon, \eta}(t) \int_{0}^{1} \frac{\partial F_{\varepsilon}}{\partial z}\left(x, t, u_{\varepsilon, \eta}(t)+\theta w_{\varepsilon, \eta}(t)\right) \mathrm{d} \theta\right) d t .
$$

Taking $x \in K_{\lambda}$, we have

$$
\left|w_{\varepsilon, \eta}(x)\right| \leq P_{K_{\lambda}, 0}\left(i_{\eta}\right)+\int_{0}^{x} m_{\lambda, \varepsilon}\left|w_{\varepsilon, \eta}(t)\right| d t .
$$


Thus, according to Gronwall's lemma, for all $x \in[-\lambda, \lambda]$,

$$
\left|w_{\varepsilon, \eta}(x)\right| \leq P_{K_{\lambda}, 0}\left(i_{\eta}\right) \exp \left(\int_{0}^{x} m_{\lambda, \varepsilon} d t\right) .
$$

Then

$$
\left|w_{\varepsilon, \eta}(x)\right| \leq P_{K_{\lambda}, 0}\left(i_{\eta}\right) \exp \left(\lambda m_{\lambda, \varepsilon}\right),
$$

and consequently

$$
P_{K_{\lambda}, 0}\left(w_{\varepsilon, \eta}\right) \leq P_{K_{\lambda}, 0}\left(i_{\eta}\right) \exp \left(\lambda m_{\lambda, \varepsilon}\right) .
$$

Then $\left(P_{K_{\lambda}, 0}\left(w_{\varepsilon, \eta}\right)\right)_{\varepsilon, \eta} \in I$. This implies the 0 th order estimate. We deduce that $\left(w_{\varepsilon, \eta}\right)_{\varepsilon, \eta} \in \mathbf{N}(\mathbf{R})$.

3.6. Independence of the generalized solution from the class of cut off functions. See $[\mathbf{8}, \mathbf{9}]$. Take $\Lambda_{1}=(0,1]$, and set

$$
\begin{aligned}
& \mathcal{X}_{1}(\mathbf{R})=\left\{\left(g_{\varepsilon}\right)_{\varepsilon} \in\left[\mathrm{C}^{\infty}(\mathbf{R})\right]^{\Lambda_{1}}: \forall K \Subset \mathbf{R}, \forall l \in \mathbf{N},\left(P_{K, l}\left(g_{\varepsilon}\right)\right)_{\varepsilon} \in|A|\right\}, \\
& \mathbf{N}_{1}(\mathbf{R})=\left\{\left(g_{\varepsilon}\right)_{\varepsilon} \in\left[\mathrm{C}^{\infty}(\mathbf{R})\right]^{\Lambda_{1}}: \forall K \Subset \mathbf{R}, \forall l \in \mathbf{N},\left(P_{K, l}\left(g_{\varepsilon}\right)\right)_{\varepsilon} \in\left|I_{A}\right|\right\}, \\
& \mathcal{A}_{1}(\mathbf{R})=\mathcal{X}_{1}(\mathbf{R}) / \mathbf{N}_{1}(\mathbf{R}) .
\end{aligned}
$$

Consider $\mathcal{T}(\mathbf{R})$ the set of families of smooth one-variable functions $\left(h_{\varepsilon}\right)_{\varepsilon \in \Lambda_{1}} \in \mathcal{X}_{1}(\mathbf{R})$, verifying the following assumptions. There exists $\left(s_{\varepsilon}\right)_{\varepsilon} \in \mathbf{R}_{*}^{(0,1]}$ :

$$
\begin{aligned}
& \sup _{z \in\left[-s_{\varepsilon}, s_{\varepsilon}\right]}\left|h_{\varepsilon}(z)\right|=1, \\
& h_{\varepsilon}(z)= \begin{cases}0 & \text { if }|z| \geq s_{\varepsilon} \\
1 & \text { if }-s_{\varepsilon}+1 \leq z \leq s_{\varepsilon}-1,\end{cases}
\end{aligned}
$$

there exists $q \in \mathbf{N}^{*}$, for all $\left(h_{\varepsilon}\right)_{\varepsilon} \in \mathcal{T}(\mathbf{R})$ and for all $\varepsilon, s_{\varepsilon} \leq r_{\varepsilon}^{q}$. Moreover, assume that $\partial^{n} h_{\varepsilon} / \partial z^{n}$ is bounded on $J_{\varepsilon}=\left[-s_{\varepsilon}, s_{\varepsilon}\right]$ for any integer $n, n>0$.

We have $\left(g_{\varepsilon}\right)_{\varepsilon \in \Lambda_{1}} \in \mathcal{T}(\mathbf{R})$. Recall that $\phi_{\varepsilon}(z)=z g_{\varepsilon}(z)$ for $z \in \mathbf{R}$, $F_{\varepsilon}(x, y, z)=F\left(x, y, \phi_{\varepsilon}(z)\right)$ for $(x, y, z) \in \mathbf{R}^{3}$ and

$$
\sup _{z \in\left[-r_{\varepsilon}, r_{\varepsilon}\right]}\left|\frac{\partial^{n} g_{\varepsilon}}{\partial z^{n}}(z)\right|=M_{n} .
$$


Let $g \in \mathcal{T}(\mathbf{R}) / \mathbf{N}_{1}(\mathbf{R})$ be the class of $\left(g_{\varepsilon}\right)_{\varepsilon}$. Take $\left(h_{\varepsilon}\right)_{\varepsilon}$ to be another representative of $g$, that is to say, $\left(h_{\varepsilon}\right)_{\varepsilon} \in \mathcal{T}(\mathbf{R})$ and

$$
\left(g_{\varepsilon}-h_{\varepsilon}\right)_{\varepsilon} \in \mathbf{N}_{1}(\mathbf{R})
$$

Set $\sigma_{\varepsilon}(z)=z h_{\varepsilon}(z)$ for $z \in \mathbf{R}, H_{\varepsilon}(x, y, z)=F\left(x, y, \sigma_{\varepsilon}(z)\right)$ for $(x, y, z) \in$ $\mathbf{R}^{3}$ and

$$
\sup _{z \in\left[-s_{\varepsilon}, s_{\varepsilon}\right]}\left|\frac{\partial^{n} h_{\varepsilon}}{\partial z}(z)\right|=M_{n}^{\prime} .
$$

Our choice is made such that $\left(\operatorname{supp}\left(h_{\varepsilon}\right)\right)_{\varepsilon}$ have the same growth as $\left(\operatorname{supp}\left(f_{\varepsilon}\right)\right)_{\varepsilon}$ with respect to the scale $\left(r_{\varepsilon}^{q}\right)_{\varepsilon}$; in this way, the corresponding solutions are lying in the same algebra $\mathcal{A}\left(\mathbf{R}^{2}\right)$.

Proposition 17. Set $S_{n}=\left\{\alpha \in \mathbf{N}^{3}:|\alpha|=n\right\}$ when $n \in \mathbf{N} *$. Let $F \in \mathrm{C}^{\infty}\left(\mathbf{R}^{3}, \mathbf{R}\right)$ and $L_{\varepsilon}$ be defined by

$$
L_{\varepsilon}(x, y, z)=F\left(x, y, \sigma_{\varepsilon}(z)\right)
$$

Assume that

$$
\begin{gathered}
\forall(x, y) \in \mathbf{R}^{2}, F(x, y, 0)=0, \\
\forall n \in \mathbf{N}, n \leq p_{0}, \exists d_{n}>0, \forall \varepsilon \in(0,1], \forall K \Subset \mathbf{R}^{2}, \\
\sup _{(x, y) \in K ; z \in J_{\varepsilon} ; \alpha \in S n}\left|D^{\alpha} F(x, y, z)\right| \leq d_{n} r_{\varepsilon}^{p_{0}}
\end{gathered}
$$

and

$$
\exists p_{0}>0, \forall \alpha \in \mathbf{N}^{3},|\alpha|=n>p_{0}, D^{\alpha} F(x, y, z)=0,
$$

then

$$
\begin{aligned}
& \forall n \in \mathbf{N}, n \leq p_{0}, \exists c_{n}>0, \forall \varepsilon \in(0,1], \forall K \Subset \mathbf{R}^{2}, \\
& \sup _{(x, y) \in K ; z \in \mathbf{R} ; \alpha \in S n}\left|D^{\alpha} L_{\varepsilon}(x, y, z)\right| \leq c_{n} r_{\varepsilon}^{p_{0}(1+q)}
\end{aligned}
$$

and $\mathcal{A}(\mathbf{R})$ is stable under the family $\left(L_{\varepsilon}\right)_{\varepsilon}$.

We refer the reader to [8] for a similar proof.

Theorem 18. Assume that $p=p_{0}(1+q)$ and the hypotheses of Proposition 17 are verified. Let $\mathcal{F}$ be the generalized operator associated 
to $F$ via the family $\left(g_{\varepsilon}\right)_{\varepsilon}$. Let $\left(h_{\varepsilon}\right)_{\varepsilon} \in\left(\mathrm{C}^{\infty}(\mathbf{R})\right)^{\Lambda_{1}}$ be another family representative of the class $\left[g_{\varepsilon}\right]=g$ and leading to another generalized operator $\mathcal{L}$ associated to $F$. Furthermore, we have $\mathcal{L}=\mathcal{F}$, i.e., $\mathcal{L}(u)=\mathcal{F}(u)$ for any $u \in \mathcal{A}(\mathbf{R})$. Then, in terms of representatives, that is to say, if $\left(u_{\varepsilon, \eta}\right)_{\varepsilon, \eta},\left(v_{\varepsilon, \eta}\right)_{\varepsilon, \eta} \in \mathcal{X}(\mathbf{R})$ and $\left(w_{\varepsilon, \eta}\right)_{\varepsilon, \eta}=\left(v_{\varepsilon, \eta}-u_{\varepsilon, \eta}\right)_{\varepsilon, \eta} \in$ $\mathbf{N}(\mathbf{R})$,

$$
\left(F\left(\cdot, \cdot, \sigma_{\varepsilon}\left(v_{\varepsilon, \eta}\right)\right)-F\left(\cdot, \cdot, \phi_{\varepsilon}\left(u_{\varepsilon, \eta}\right)\right)\right)_{\varepsilon, \eta} \in \mathbf{N}(\mathbf{R}) .
$$

We refer the reader to $[\mathbf{8}]$ for a similar proof.

Corollary 19. Problem $\left(P_{\text {gen }}\right)$ and, a fortiori, its solution, does not depend of the choice of the representative $\left(f_{\varepsilon}\right)_{\varepsilon}$ of the class $f \in$ $\mathcal{T}(\mathbf{R}) / \mathbf{N}_{1}(\mathbf{R})$.

\subsection{Examples.}

Example 20. Consider the equation

$$
(P): u(x)=L(x)-\int_{0}^{x} u^{2}(t) d t
$$

This equation is equivalent to the nonlinear differential Ricatti equation:

$$
\left\{\begin{array}{l}
u^{\prime}(x)+u^{2}(x)=l(x) \\
u(0)=L(0) ; l=L^{\prime}
\end{array}\right.
$$

In $[\mathbf{1 2}]$ the authors show that, under some conditions, this equation where $l$ and $u$ are elements from Colombeau algebra $\mathcal{G}(\mathbf{R})$ with $u(0)=$ $L(0)$ a known generalized number, has a unique solution in $\mathcal{G}(\mathbf{R})$.

Example 21. Consider the equation

$$
(P): u(x)=c+\int_{0}^{x}\left(u-u^{2}\right)(t) d t
$$

where $c$ is a generalized number $c=\left[c_{\varepsilon, \eta}\right] \in \overline{\mathbf{R}}$ (the algebra of generalized numbers). This equation is equivalent to the logistic equation

$$
u^{\prime}(x)=u(x)-u^{2}(x) ; \quad u(0)=c .
$$


Using our previous notation, we have $F_{\varepsilon}\left(x, t, u_{\varepsilon, \eta}(t)\right)=\phi_{\varepsilon}\left(u_{\varepsilon, \eta}(t)\right)-$ $\left(\phi_{\varepsilon}\left(u_{\varepsilon, \eta}(t)\right)\right)^{2}$ and the family $\left(P_{(\varepsilon, \eta)}\right)$ of regularized problems:

$$
\left(P_{(\varepsilon, \eta)}\right): u_{\varepsilon, \eta}(x)=c_{\varepsilon, \eta}+\int_{0}^{x} F_{\varepsilon}\left(x, t, u_{\varepsilon, \eta}(t)\right) d t,
$$

is associated to problem $(P)$. The solution $u_{\varepsilon, \eta}$ to problem $\left(P_{(\varepsilon, \eta)}\right)$ is defined by

$$
u_{\varepsilon, \eta}(x)=\frac{c_{\varepsilon, \eta} e^{x}}{1+c_{\varepsilon, \eta}\left(e^{x}-1\right)} .
$$

So $u=\left[u_{\varepsilon, \eta}\right]$ is the solution to Problem $(P), u$ belongs to $\mathcal{A}(\mathbf{R})$ and $u$ is not a distribution.

Example 22. In the case of linear equations, we recover well-known results. We use one parameter $\eta$ to regularize the data, then we obtain the Colombeau algebra. Consider the equation

$$
(P): u(x)=G(x)+\int_{0}^{x} u(t) d t
$$

it is equivalent to the differential equation

$$
u^{\prime}(x)=g(x)+u(x) ; \quad u(0)=G(0)
$$

where $G^{\prime}=g$.

Let $H$ be the Heaviside distribution. Then $G$ is the distribution $H * g$ so the distributional solution is $u=E * g$ with $E(t)=H(t) e^{t}$. If $\left(\theta_{\eta}\right)_{\eta \in(0,1]}$ is a family of mollifiers, the family of problems

$$
\left(P_{(\eta)}\right): u_{\eta}(x)=g_{\eta}(x)+\int_{0}^{x} u(t) d t
$$

with $g_{\eta}=\left(G * \theta_{\eta}\right)$, is associated to problem $(P)$. Then

$$
u_{\eta}(x)=\int_{0}^{x} g_{\eta}(t) e^{x-t} d t
$$

and, as $\left[g_{\eta}\right] \backsim g$, we have $u=\left[u_{\eta}\right] \backsim E * g$. In particular, take $G=H$. Then $g=\delta$ and $u \backsim E * \delta=E$ so $u(x)=H(x) e^{x}$. 
4. Consistency with classical results. Suppose that $\varphi \in \mathrm{C}^{\infty}(\mathbf{R})$ then $\mathcal{C}=A / I_{A}$ is overgenerated by the following elements of $\mathbf{R}_{*}^{(0,1]}$ : $(\varepsilon)_{\varepsilon},\left(r_{\varepsilon}\right)_{\varepsilon},\left(\exp r_{\varepsilon}\right)_{\varepsilon} \cdot \mathcal{A}(\mathbf{R})=\mathcal{X}(\mathbf{R}) / \mathbf{N}(\mathbf{R})$ is built on $\mathcal{C}$. We have the same results as previously taking $\varphi_{\eta}=\varphi$.

Remark 23. The generalized function represented by the family of solutions to the regularized problems $\left(P_{\varepsilon}\right)$ is defined from the integral representation $u_{\varepsilon}(x)=\varphi(x)+\int_{0}^{x} F_{\varepsilon}\left(x, t, u_{\varepsilon}(t)\right) d t$. Thus, we are going to study the relationship between this generalized function and the classical solutions to $(P)$ (when they exist) on a domain $\Omega=] \mu, \nu[$. If Problem $(P)$ has a smooth solution $v$ on $\Omega$ then, necessarily we have $\Omega \subset \mathbf{R} \backslash \operatorname{singsupp}(u)$.

Recall that there exists a canonical sheaf embedding of $\mathrm{C}^{\infty}$ into $\mathcal{A}$, through the morphism of algebra

$$
\sigma_{O}: \mathrm{C}^{\infty}(O) \longrightarrow \mathcal{A}(O), \quad g \longmapsto\left[g_{\varepsilon}\right]
$$

(where $O$ is any open subset of $R$ and $g_{\varepsilon}=g$ ).

The presheaf $\mathcal{A}$ allows to define restriction and, as usual, we denote by $\left.u\right|_{O}$ the restriction on $O$ of $u \in \mathcal{A}(\mathbf{R})$.

Theorem 24. Let $\Omega=] \mu, \nu[$ be an open subset of $\mathbf{R}$ such that

$$
\Omega \subset \mathbf{R} \backslash \operatorname{singsupp}(u) \text {. }
$$

Assume that $\Omega=\cup_{\varepsilon} \Omega_{\varepsilon}$ when $\left(\Omega_{\varepsilon}\right)_{\varepsilon}$ is an increasing family of open subsets of $\mathbf{R}$ such that $\left.\Omega_{\varepsilon}=\right] \mu_{\varepsilon}, \nu_{\varepsilon}\left[\right.$ with $\mu_{\varepsilon}<\nu_{\varepsilon}$. Assume that the nonregularized problem has a smooth solution $v$ on $\Omega$ such that $\sup _{x \in \Omega_{\varepsilon}}|v(x)|<r_{\varepsilon}-1$ for any $\varepsilon$. Let $u \in \mathcal{A}(\mathbf{R})$ be the generalized function represented by the family $\left(u_{\varepsilon}\right)_{\varepsilon}$ of solutions to $\left(P_{\varepsilon}\right)$ given by Theorem 16. Then $\sigma_{\Omega}(v)=\left.u\right|_{\Omega}$.

Proof. See $[\mathbf{5}, \mathbf{7}, \mathbf{9}]$. We can choose as representative of $\sigma_{\Omega}(v)$ the net $(v)_{\varepsilon}$. We clearly have for all $x \in \Omega$, there exists a $\varepsilon_{0}$, for all $\varepsilon \leq \varepsilon_{0}$, $x \in \Omega_{\varepsilon}$. Then

$$
v(x)=\varphi(x)+\int_{0}^{x} F(x, t, v(t)) d t .
$$


We take as representative of $u$ the net $\left(u_{\varepsilon}\right)_{\varepsilon}$ given by Theorem 16 . This net satisfies for all $x \in \Omega$,

$$
u_{\varepsilon}(x)=\varphi(x)+\int_{0}^{x} F_{\varepsilon}\left(x, t, u_{\varepsilon}(t)\right) d t .
$$

Take $K \Subset \Omega$. There exists a $\varepsilon_{1}$ such that, for all $\varepsilon \leq \varepsilon_{1}, K \Subset \Omega_{\varepsilon}$. According to the definition of $\Omega_{\varepsilon}$, there exists a $\lambda, 0<\lambda<\left(\nu_{\varepsilon}-\mu_{\varepsilon}\right) / 2$, such that $K \subset Q_{\lambda} \subset \Omega_{\varepsilon}$ with $Q_{\lambda}=\left[\mu_{\varepsilon}+\lambda, \nu_{\varepsilon}-\lambda\right]$. Note that, for $\left.(x, t, z) \in \Omega_{\varepsilon}^{2} \times\right]-r_{\varepsilon}+1, r_{\varepsilon}-1\left[\right.$, we have $F(x, t, z)=F_{\varepsilon}(x, t, z)$ by construction of $F_{\varepsilon}$. Set $\left(w_{\varepsilon}\right)_{\varepsilon}=\left(v-\left.u_{\varepsilon}\right|_{\Omega}\right)_{\varepsilon}$. Take $x \in K$. Then

$$
\begin{aligned}
w_{\varepsilon}(x) & =\int_{0}^{x} F(x, t, v(t)) d t-\int_{0}^{x} F_{\varepsilon}\left(x, t, u_{\varepsilon}(t)\right) d t \\
& =\int_{0}^{x}\left(F(x, t, v(t))-F_{\varepsilon}(x, t, v(t))\right) d t \\
& +\int_{0}^{x}\left(F_{\varepsilon}(x, t, v(t))-F_{\varepsilon}\left(x, t, u_{\varepsilon}(t)\right)\right) d t
\end{aligned}
$$

However,

$$
\begin{aligned}
F_{\varepsilon}(x, t, v(t))-F_{\varepsilon}\left(x, t, u_{\varepsilon}(t)\right) \\
\quad=\left(v(t)-u_{\varepsilon}(t)\right)\left(\int_{0}^{1} \frac{\partial F_{\varepsilon}}{\partial z}\left(x, t,\left(u_{\varepsilon}(t)+\sigma(v(t))-u_{\varepsilon}(t)\right)\right) d \sigma\right) .
\end{aligned}
$$

Thus,

$$
\left|F_{\varepsilon}(x, t, v(t))-F_{\varepsilon}\left(x, t, u_{\varepsilon}(t)\right)\right| \leq\left|w_{\varepsilon}(t)\right| m_{Q_{\lambda}, \varepsilon} .
$$

Moreover, as the values of $v$ are in $]-r_{\varepsilon}+1, r_{\varepsilon}-1[$, we have

$$
F(\cdot, \cdot, v)-F_{\varepsilon}(\cdot, \cdot, v)=0
$$

on $\Omega_{\varepsilon}$. So

$$
\left|w_{\varepsilon}(x)\right| \leq m_{Q_{\lambda}, \varepsilon} \int_{0}^{x}\left|w_{\varepsilon}(t)\right| d t .
$$

Thus, according to Gronwall's lemma, $w_{\varepsilon}(x)=0$; hence, $w_{\varepsilon}=0$ on $K$. It follows that, for all $\varepsilon \leq \varepsilon_{1}, \sup _{x \in Q_{\lambda}}\left|w_{\varepsilon}(x)\right|=0$; hence, $\left(P_{K, l}\left(w_{\varepsilon}\right)\right)_{\varepsilon} \in$ $I_{A}$ for any $l \in \mathbf{N}$ as $w_{\varepsilon}$ vanishes on $K$. Thus, $\left(w_{\varepsilon}\right)_{\varepsilon} \in \mathbf{N}(\Omega)$ and $\sigma_{\Omega}(v)=\left.u\right|_{\Omega}$ as claimed. 
Remark 25. The hypotheses made in Theorem 24 are satisfied for $F(\cdot, \cdot, u)=u^{k}(k \geq 2)$ and $\varphi=1$, for which the local solution

$$
v(x)=(1-(k-1) x)^{-(k-1)^{-1}}
$$

exists in $]-\infty,(k-1)^{-1}\left[\right.$. On $\left.\Omega_{\varepsilon}=\right]-\infty,(1-\varepsilon)(k-1)^{-1}[$, we have $|u(x)| \leq \varepsilon^{-(k-1)^{-1}}$. It suffices to take $r_{\varepsilon}>\varepsilon^{-(k-1)^{-1}}$, say $r_{\varepsilon}>\varepsilon^{-1}[\mathbf{5}]$.

Let $\left(u_{\varepsilon}\right)_{\varepsilon}$ be the family of solutions to $\left(P_{\varepsilon}\right)$. Then $u=\left[u_{\varepsilon, \eta}\right]_{\mathcal{A}(\mathbf{R})}$ is the solution to $\left(P_{\text {gen }}\right)$. The theorem shows that the restriction of $u \in \mathcal{A}\left(\mathbf{R}^{2}\right)$ to $\Omega$ is precisely $v$. The local classical solution $v$, which blows up for $x=(k-1)^{-1}$, extends to a global generalized solution $u$ which absorbs this blow up.

Take $k=2$. Then $v(x)=(1-x)^{-1}$ and the generalized solution is $u \sim v p\left((1-x)^{-1}\right)$ where $v p$ denotes the principal value distribution.

\section{APPENDIX}

We use the method of successive approximations to establish the existence and uniqueness of a solution of the integral equation:

$$
u(x)=\varphi(x)+\int_{0}^{x} F(x, t, u(t)) d t
$$

when the integrand $F$ satisfies a Lipschitz condition as a function of its third argument $u$.

We define a sequence of successive approximations for the solution of the integral equation by means of the formula:

$$
u_{n+1}(x)=u_{0}(x)+\int_{0}^{x} F\left(x, t, u_{n}(t)\right) d t .
$$

The first approximation $u_{0}$ will be taken here to be $u_{0}(t)=\varphi(x)$. Our purpose is to establish that, under suitable hypotheses about the integrand $F$, the limit

$$
u=u_{0}+\lim _{n \rightarrow+\infty} u_{n}=u_{0}+\sum_{n=0}^{\infty}\left(u_{n+1}-u_{n}\right)
$$


of the successive approximations exists and satisfies the integro-differential equation (ivp).

For the proof of convergence of the infinite series we shall need to estimate differences corresponding to increments in the argument $z$.

Let $K$ be a compact of $\mathbf{R}$ and there exists a $\lambda>0$ such that $K \subset K_{\lambda}=[-\lambda,+\lambda]$.

For any $(x, t) \in K_{\lambda}^{2}$, the mean value theorem yields the expression

$$
F(x, t, z)-F(x, t, r)=(z-r) \int_{0}^{1} \frac{\partial F}{\partial z}(x, t, r+\sigma(t-r)) d \sigma .
$$

According to hypothesis $(H)$, we put

$$
m_{\lambda}=\sup _{(x, t) \in K_{\lambda}^{2} ; z \in \mathbf{R}}\left|\frac{\partial F}{\partial z}(x, t, z)\right| .
$$

We can conclude that $|F(x, t, z)-F(x, t, r)| \leq m_{\lambda}|z-r|$.

In particular, $\left|F\left(x, t, u_{0}(t)\right)-F(x, t, 0)\right| \leq m_{\lambda}\left|u_{0}(t)\right|$. Then

$$
\left|F\left(x, t, u_{0}(t)\right)\right| \leq|F(x, t, 0)|+m_{\lambda}\left|u_{0}(t)\right| .
$$

Set

$$
\Phi_{\lambda}=\sup _{(x, t) \in K_{\lambda}^{2}}|F(x, t, 0)|+m_{\lambda} \sup _{x \in K_{\lambda}}|\varphi(x)| .
$$

We have for all $x \in K_{\lambda}$,

$$
\left|u_{n+1}(x)-u_{n}(x)\right| \leq \int_{0}^{x} m_{\lambda}\left|u_{n}(t)-u_{n-1}(t)\right| d t
$$

We have

$$
u_{1}(x)-u_{0}(x)=\int_{0}^{x} F\left(x, t, u_{0}(t)\right) d t
$$

thus,

$$
\left|u_{1}(x)-u_{0}(x)\right| \leq \int_{0}^{x} \Phi_{\lambda} d t \leq x \Phi_{\lambda}
$$

So we find that

$$
\begin{aligned}
\left|u_{2}(x)-u_{1}(x)\right| & \leq \int_{0}^{x} m_{\lambda}\left|u_{1}(t)-u_{0}(t)\right| d t \\
& \leq m_{\lambda} \Phi_{\lambda} \int_{0}^{x} t d t \leq m_{\lambda} \Phi_{\lambda} \frac{x^{2}}{2} .
\end{aligned}
$$


By induction, we obtain:

$$
\left|u_{n+1}(x)-u_{n}(x)\right| \leq \frac{\Phi_{\lambda}}{m_{\lambda}} \frac{\left(m_{\lambda} x\right)^{n+1}}{(n+1) !} .
$$

Thus,

$$
\frac{\Phi_{\lambda}}{m_{\lambda}} e^{m_{\lambda} x}=\frac{\Phi_{\lambda}}{m_{\lambda}} \sum_{n=0}^{\infty} \frac{\left(m_{\lambda} x\right)^{n+1}}{(n+1) !}
$$

is a majorant for the infinite series $\sum_{n=0}^{\infty}\left\|u_{n+1}-u_{n}\right\|_{\infty, K_{2, \lambda}}$, and it follows that the series $\sum_{n=0}^{\infty}\left(u_{n+1}-u_{n}\right)$ converges uniformly on any compact of $\mathbf{R}$. For any $n, u_{n}$ is continuous; thus, $u-u_{0}=$ $\sum_{n=0}^{\infty}\left(u_{n+1}-u_{n}\right)$ is continuous on $\mathbf{R}$.

We obtain the estimate

$$
\|u\|_{\infty, K} \leq\|u\|_{\infty, K_{\lambda}} \leq\left\|u_{0}\right\|_{\infty, K_{\lambda}}+\frac{\Phi_{\lambda}}{m_{\lambda}} e^{\lambda m_{\lambda}} .
$$

We compute

$$
\begin{aligned}
u_{n}(t)-\left(u_{0}(t)+\int_{0}^{x} F(x, t\right. & , u(t)) d t) \\
& =\int_{0}^{x}\left(F\left(x, t, u_{n}(t)\right)-F(x, t, u(t))\right) d t
\end{aligned}
$$

and

$$
\left|\int_{0}^{x}\left(F\left(x, t, u_{n}(t)\right)-F(x, t, u(t))\right) d t\right| \leq m_{\lambda}\left|u_{n}(t)-u(t)\right| .
$$

We have

$$
\begin{aligned}
u(t)- & \left(u_{0}(t)+\int_{0}^{x} F(x, t, u(t)) d t\right) \\
& =\left(u(t)-u_{n}(t)\right)+\left[u_{n}(t)-\left(u_{0}(t)+\int_{0}^{x} F(x, t, u(t)) d t\right)\right] ;
\end{aligned}
$$

thus

$$
\begin{aligned}
\mid u(t)-\left(u_{0}(t)+\int_{0}^{x} F(\right. & x, t, u(t)) d t) \mid \\
& \leq\left|u_{n}(t)-u(t)\right|+m_{\lambda} \int_{0}^{x}\left|u_{n}(t)-u(t)\right| d t \\
& \leq\left|u_{n}(t)-u(t)\right|\left(1+\lambda m_{\lambda}\right) .
\end{aligned}
$$


We conclude that, for any $x \in K_{\lambda}$,

$$
u(x)=u_{0}(x)+\int_{0}^{x} F(x, t, u(t)) d t .
$$

Let us show the uniqueness of the solution. Let $v$ be another solution; putting $\Delta=v-u$, we obtain

$$
\Delta(x)=\int_{0}^{x}(F(x, t, v(t))-F(x, t, u(t))) d t .
$$

We have

$$
\begin{aligned}
|\Delta(x)| & \leq \int_{0}^{x}(F(x, t, v(t))-F(x, t, u(t))) d t \\
& \leq m_{\lambda} \int_{0}^{x}|v(t)-u(t)| d t \leq m_{\lambda} \int_{0}^{x}|\Delta(t)| d t .
\end{aligned}
$$

By applying Gronwall's lemma, we get $\Delta=0$, which proves the uniqueness of $u$.

\section{REFERENCES}

1. S. Bernard, J.-F. Colombeau and A.Delcroix, Generalized integral operators and applications, Math. Proc. Cambr. Philos. Soc. 141 (2006), 521-546.

2. J.-F. Colombeau, Elementary introduction to new generalized functions, North Holland Math. Stud. 113, North-Holland, Amsterdam, 1984.

3. - New generalized functions and multiplication of distributions, NorthHolland, Amsterdam, 1984.

4. A. Delcroix, Remarks on the embedding of spaces of distributions into spaces of Colombeau generalized functions, Novisad J. Math. 35 (2005), 27-40.

5. A. Delcroix, V. Dévoué and J.-A. Marti, Generalized solutions of singular differential problems. Relationship with classical solutions, J. Math. Anal. Appl. 353 (2009), 386-402.

6. — Well posed differential problems in algebras of generalized functions, Appl. Anal. 90 (2011), 1747-1761.

7. V. Dévoué, Generalized solutions to a non Lipschitz Cauchy problem, J. Appl. Anal. 15 (2009), 1-32.

8. - Generalized solutions to a non Lipschitz Goursat problem, Diff. Eq. Appl. 1 (2009), 153-178.

9. - Generalized solutions to a singular nonlinear Cauchy problem, Novi Sad J. Math. 41 (2011), 85-121. 
10. C. Garetto, Topological structures in Colombeau algebras II: Investigation into the duals of $\mathcal{G}_{c}(\Omega), \mathcal{G}(\Omega)$ and $\mathcal{G}_{s}\left(\mathbf{R}^{n}\right)$, Monatsh. Math. 146 (2005), 203-226.

11. M. Grosser, M. Kunzinger, M. Oberguggenberger and R. Steinbauer, Geometric theory of generalized functions with applications to general relativity, Kluwer Academic Press, Dordrecht, 2001.

12. B. Jolevska-Tuneska, A. Takači and E. ÖzCcag், On differential equations with nonstandard coeffients, Appl. Anal. Dis. Math. 1 (2007), 276-283.

13. J.-A. Marti, Fundamental structures and asymptotic microlocalization in sheaves of generalized functions, Int. Transforms Spec. Funct. 6 (1998), 223-228.

14. -,$(\mathcal{C}, \mathcal{E}, \mathcal{P})$-Sheaf structures and applications, in Nonlinear theory of generalized functions, M. Grosser et al., eds., Chapman \& Hall/CRC, Boca Raton, 1999.

15. - Multiparametric algebras and characteristic cauchy problem, in Nonlinear algebraic analysis and applications, Proc. Inter. Conf. Generalized Functions (ICGF 2000). CSP (2004), 181-192.

16. Non linear algebraic analysis of delta shock wave solutions to burger's equation, Pacific J. Math. 210 (2003), 165-187.

17. M. Oberguggenberger, Multiplication of distributions and applications to partial differential equations, Pitman Res. Notes Math. 259, Longman, Harlow, 1992 .

18. S. Pilipović and M. Stojanović, Generalized solutions to nonlinear Volterra integral equations with non-Lipschitz nonlinearity, Nonlinear Anal. 37 (1997), 319-335.

19. D. Scarpalézos, Colombeau's generalized functions: Topological structures; Microlocal properties. A simplified point of view, Part I, Bull. Cl. Sci. Math. Nat. Sci. Math. 25 (2000), 89-114.

20. - Colombeau's generalized functions: Topological structures; Microlocal properties. A simplified point of view, Part II, Publ. Inst. Math. (Beograd) 76 (2004), 111-125.

Equipe Analyse Algébrique Non Linéaire, Laboratoire CEREGMiA, Université des Antilles et de la Guyane, Campus de Schoelcher, BP 7209 , 97275 Schoelcher Cedex, Martinique, F.W.I.

Email address: devoue-vi@orange.fr 